\title{
PRÁTICAS DE BOM GOVERNO NAS EMPRESAS DO SETOR PÚBLICO EM PORTUGAL
}

\author{
Ana Paula Ferreira ${ }^{1}$ e Ana Lúcia Romão ${ }^{12}$ \\ ${ }^{1}$ Instituto Superior de Ciências Sociais e Políticas, Portugal. apferreira@iscsp.ulisboa.pt; anaromao@iscsp.ulisboa.pt; \\ ${ }^{2}$ Centro de Administração e Políticas Públicas, Portugal
}

\begin{abstract}
Resumo. Esta pesquisa tem como objetivo explorar o conceito e as diferentes perspetivas de Responsabilidade Social nas empresas do setor público em Portugal, dada a sua relevância na atividade económica nacional. Propõe-se a realização de um diagnóstico das práticas e da respetiva divulgação nestas empresas, no que se refere a questões ambientais, sociais e de governança. O modelo de avaliação, assente na análise de conteúdo das diversas tipologias de informação apresentada nas páginas web das empresas, pressupõe duas etapas distintas. A primeira etapa, a partir da atribuição de scores, permite avaliar o grau de cumprimento das empresas em matérias de divulgação, bem como o nível de transparência. A segunda etapa, a partir da construção de índices por grupo, irá permitir avaliar a qualidade da informação disponibilizada. Concluiu-se que uma parte significativa das empresas não divulga toda a informação legalmente exigida nos seus sites, o acesso nem sempre é direto e, muitas vezes, encontra-se desatualizada. Algumas empresas do setor público não estão a contribuir para a boa implementação das políticas públicas nem para a disseminação de boas práticas nos domínios económico, social e ambiental, não podendo ser consideradas como um bom exemplo na divulgação destas questões.
\end{abstract}

Palavras-chave: Análise de Conteúdo; Divulgação online; Responsabilidade Social das Empresas; Setor Público Empresarial; Transparência.

\section{GOOD GOVERNANCE PRACTICES IN STATE-OWNED ENTERPRISES IN PORTUGAL}

\begin{abstract}
This research aims to explore the concept and the different perspectives of Social Responsibility in state-owned enterprises in Portugal, given its relevance in national economic activity. A diagnosis of the practices and the respective disclosure in these companies was carried out, with regard to environmental, social and governance issues. The evaluation model, based on content analysis of the different types of information disclosed on the companies' web pages, assumes two distinct stages. For the first stage, the attribution of scores, allow to estimate the degree of compliance of companies in matters of disclosure, as well as the level of transparency. The second stage, based on the construction of indexes by group, will allow evaluating the quality of the information disclosed. It was concluded that a significant part of companies do not disclose all the information legally required on their websites, access is not always direct and, often, is out of date. Some of the public sector companies are not contributing to the good implementation of public policies or to the dissemination of good practices in the economic, social and environmental fields, so for that they cannot be considered as a good example in the dissemination of these issues.
\end{abstract}

Keywords: Content Analysis; Corporate Social Responsibility; Online Disclosure; State-owned Enterprises; Transparency.

\section{INTRODUÇÃO}

Esta pesquisa tem como objetivo explorar o conceito e as diferentes perspetivas de Responsabilidade Social das Empresas (RSE), no contexto das políticas públicas. A contribuição principal do estudo consiste na realização de um diagnóstico abrangente das práticas e da respetiva divulgação, no que se refere a questões ambientais, sociais e de 
governança (ESG), das empresas do setor público em Portugal. O setor público empresarial representa uma parte importante da atividade económica nacional e o seu contributo para a boa implementação das políticas públicas é fundamental.

O papel económico e social destas empresas é da maior relevância, e a existência de práticas de bom governo contribui para o bom desempenho das entidades e para a disseminação de boas práticas nesta matéria. Quanto às ações de boas práticas nos domínios económico, social e ambiental, estas entidades deveriam dar o exemplo na divulgação destas questões, em alinhamento com as recomendações de boas práticas internacionais, nomeadamente no que tange às Diretrizes da OCDE de Governança Corporativa para Empresas Estatais (OCDE, 2018).

O facto de não existir um modelo único para a divulgação da informação de responsabilidade social das empresas públicas, e a dificuldade de avaliação que daí advém, contribui para o especial interesse do modelo de avaliação que se propõe. A metodologia apresentada incide na combinação de matrizes de análise de conteúdo que permitem validar a qualidade da divulgação respeitante às práticas ESG e determinar o índice de transparência das empresas, podendo vir a ser replicada noutros estudos análogos.

\section{ENQUADRAMENTO TEÓRICO}

O quadro teórico do estudo assentará na exploração do conceito e nas diferentes perspetivas de Responsabilidade Social nas empresas do setor público em Portugal. A Comissão Europeia define a RSE como "a responsabilidade das empresas pelos seus impactos na sociedade" (COM (2011) 681, p. 6). Carroll (2015) aponta para uma evolução do conceito, que se estendeu a outras áreas e responsabilidades com base nas ações "proteger" e "melhorar", implicando que as empresas devem evitar ou reduzir os seus impactos negativos (por exemplo, poluição, discriminação, corrupção, etc.).

Os conceitos de accountability e transparência, muito relacionados entre si, darão igualmente suporte teórico à exploração do material empírico. O termo accountability, com um significado bastante amplo (Bovens, Schillemans, \& Hart, 2008), contempla as exigências de informação por parte dos cidadãos e a transparência refere-se ao acesso à informação. Ambos os conceitos remetem para o cabal cumprimento das obrigações de divulgação de informação das instituições públicas. A transparência favorece a prevenção da corrupção, promove a eficiência, a integridade e a accountability, para além de reforçar a 
confiança dos cidadãos na administração pública. A transparência permite também melhorar a qualidade das políticas públicas e a qualidade de vida dos cidadãos, condições fundamentais para o bom funcionamento das democracias.

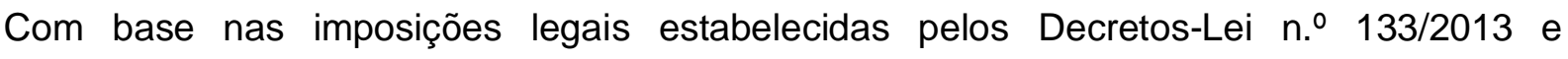
n. $.89 / 2017$, propomos um modelo de análise que permita avaliar a eficácia dos diplomas legais em vigor, bem como do contributo destas empresas para o fortalecimento da política pública.

O Decreto-Lei n. 133/2013 estabelece os princípios e regras aplicáveis ao sector público empresarial e cria a Unidade Técnica de Acompanhamento e Monitorização do Sector Público Empresarial (UTAM). Este diploma pretende estabelecer um regime jurídico mais exigente, mas também mais claro, transparente e eficaz, no que respeita ao controlo da legalidade e da boa gestão pública na alocação de recursos públicos para a prossecução de atividades em modo empresarial. Assim, interessa averiguar, no âmbito dos princípios de governo societário, as práticas de bom governo destas empresas, nomeadamente quanto às obrigações de divulgação de informação, transparência, prevenção da corrupção, padrões

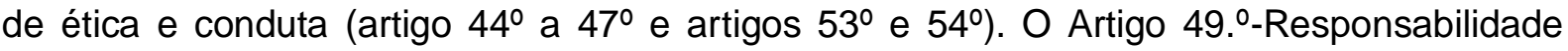
social, exige que as empresas públicas prossigam objetivos de responsabilidade social e ambiental, proteção dos consumidores, investimento na valorização profissional, promoção da igualdade e não discriminação, proteção do ambiente e respeito por princípios de legalidade e ética empresarial.

O Decreto-Lei n.. 89/2017, que resulta da transposição da Diretiva $n .{ }^{\circ}$ 2014/95/UE, obriga as grandes empresas que sejam entidades de interesse público e que tenham em média mais de 500 trabalhadores, a incluir no seu relatório de gestão uma demonstração não financeira. Esta informação deverá permitir a compreensão da evolução, do desempenho, da posição e do impacto das suas atividades, referentes, no mínimo, às questões ambientais, sociais e relativas aos trabalhadores, à igualdade entre mulheres e homens, à não discriminação, ao respeito dos direitos humanos, ao combate à corrupção e às tentativas de suborno, incluindo: a) Uma breve descrição do modelo empresarial da empresa; b) Uma descrição das políticas seguidas pela empresa em relação a essas questões, incluindo os processos de diligência devida aplicados; c) Os resultados dessas políticas; d) Os principais riscos associados a essas questões, ligados às atividades da empresa, incluindo, se relevante e proporcionado, as suas relações empresariais, os seus produtos ou serviços suscetíveis de 
ter impactos negativos nesses domínios e a forma como esses riscos são geridos pela empresa; e) Indicadores-chave de desempenho relevantes para a sua atividade específica.

Este novo contexto favoreceu o debate sobre as responsabilidades das empresas em relação às questões ESG. As práticas de RSE nas empresas do setor público começam a emergir, em consonância com o novo paradigma e a estrutura legal recente.

\section{METODOLOGIA}

Este estudo propõe um modelo de avaliação com recurso à análise documental e à análise de conteúdo, que incide sobre a informação apresentada nas páginas web, pelas empresas do setor público. Incorpora uma dimensão exploratória, que procura "descobrir" o que as empresas têm vindo a divulgar no âmbito da RSE, bem como uma dimensão mais sistematizada que permitirá a verificação da sua confirmação ou infirmação, e que "servirá de prova" para as evidências (Bardin, 2016).

A amostra em estudo foi obtida a partir da listagem de empresas públicas do setor empresarial do estado disponibilizada pela UTAM e atualizada a 30/04/2019, onde constam 188 empresas e estão incluídos 13 setores de atividade. A amostra foi selecionada de forma a incluir pelo menos duas representações por cada setor de atividade.

O desenho do modelo de pesquisa foi suportado no referencial teórico e noutros estudos que utilizaram a metodologia da análise de conteúdo dos relatórios de gestão e outras informações disponíveis online (Bonsón \& Bednárová, 2015; Faria, 2017; Kumar \& Kidwai, 2018; Sánchez, Bolívar \& Hernández, 2015; Venturelli, Caputo, Leopizzi \& Pizzi, 2019). O modelo assenta na análise de conteúdo realizada em duas etapas distintas, recorrendo a procedimentos sistemáticos e objetivos, à enumeração, à descrição e à interpretação do conteúdo das mensagens, obtendo-se indicadores (qualitativos e/ou quantitativos) que permitem a inferência e a significação a partir da leitura dos dados (Bardin, 2016), com recurso a software de análise de dados qualitativos (CAQDAS). O modelo que se propõe pretende servir de base para investigações futuras aplicadas a outras amostras.

$\mathrm{Na}$ primeira etapa foram identificadas as tipologias de informação, selecionadas a partir da sobreposição entre as exigências legais e as recomendações do Global Reporting Initiative (GRI), que se encontravam disponíveis a partir da homepage de cada uma das empresas e, ainda, as que se encontravam inseridas noutros documentos. A sistematização da recolha 
de informação foi conseguida com recurso a uma matriz de cariz dicotómico na qual se identificava a disponibilidade, o acesso e ainda a atualidade do item para os casos em que esta se justifica. Cada uma destas entradas foi pontuada com 0 ou 1 em função da ausência ou presença da informação, se a informação se encontrava disponível no contexto de outros documentos (acesso indireto - 0) ou se era divulgada em documento autónomo com link próprio (acesso direto - 1) e ainda se estava ou não atualizada.

No Quadro 1 são apresentadas as tipologias de informação que se entendeu serem relevantes para a avaliação da divulgação, no que se refere à ESG, e que são simultaneamente obrigatórias por lei. Esta primeira fase de análise permitiu avaliar o grau de cumprimento das empresas em matérias de divulgação, bem como o nível de transparência. A determinação do nível de transparência é realizada com a contribuição equitativa dos 10 itens em análise.

A segunda etapa do estudo consiste numa análise qualitativa do conteúdo, assente na análise conceptual e temática, incidindo nos relatórios de gestão (relatório e contas e/ou relatório societário) e sustentabilidade das empresas, assim como noutras informações que se consideraram relevantes e que se encontravam disponibilizadas nas páginas web. Para esta fase, ainda em curso, a opção recaiu sobre um modelo dedutivo, onde a construção do sistema de categorias foi realizada numa combinação de processos apriori, definindo uma matriz de categorias e subcategorias que serviu de ponto de partida para a análise, e processos aposteriori, que, no decorrer da exploração qualitativa, deixaram em aberto a possibilidade de se poder vir a encontrar novas subcategorias de análise (Bardin, 2016; Vala, 1986). As categorias representam as diversas dimensões da RSE e assentam na revisão da literatura, na legislação em vigor e nas recomendações das organizações internacionais (Quadro 2). A codificação do material, em cada categoria, foi realizada previamente, mas com a possibilidade de integração de novos códigos que possam vir a dar maior consistência e diversidade à exploração do texto. As unidades de análise, consideradas válidas, pertinentes e/ou adequadas para os objetivos, de natureza interpretativa, assentam nas ideias-chave dentro de cada categoria. Aos grupos de informação considerados foram atribuídos scores de acordo com o tipo de informação: ausência - 0; narrativa -1 ; quantitativa -2 ; narrativa + quantitativa -3 . Estes scores contribuem para a construção dos respetivos índices por grupo, permitindo desta forma avaliar a qualidade da informação disponibilizada. Assim, é possível construir a partir da avaliação de cada grupo um índice que reflete a posição de cada empresa em relação à 
RSE. Foi ainda avaliada de forma qualitativa, a existência de impacto positivo das empresas sobre a sociedade de uma forma geral e nas suas relações diretas para cada um dos grupos de informação considerados na análise. A classificação foi atribuída da seguinte forma: ausência -0 ; relevante -1 ; muito relevante -2 .

Quadro 1. Conteúdos de divulgação obrigatória previstos no DR n.ำ133/2017

Declaração de missão, visão e valores
Organograma
Identidade dos órgãos sociais ou estatutários
Apresentação dos elementos curriculares de todos os membros dos órgãos sociais ou estatutários
Código de ética e conduta
Plano de atividades
Plano de prevenção de riscos de corrupção e infrações conexas
Relatório e contas
Relatório societário
Relatório de sustentabilidade

Quadro 2. Lista de conteúdos de RSE (em construção)

\begin{tabular}{l|l}
\hline Categoria & Subcategoria \\
\hline \multirow{3}{*}{ Ambiente } & $\begin{array}{l}\text { Ações de cariz ambiental } \\
\text { Gestão de resíduos } \\
\text { Redução do consumo de água } \\
\text { Redução do consumo de energia }\end{array}$ \\
\hline \multirow{3}{*}{ Social } & $\begin{array}{l}\text { Equidade de condições por género nos colaboradores } \\
\text { Formação profissional } \\
\text { Igualdade de género nos órgãos sociais } \\
\text { Prazo de pagamento a fornecedores }\end{array}$ \\
\hline \multirow{3}{*}{ Governança } & $\begin{array}{l}\text { Avaliação do governo societário } \\
\text { Divulgação das ocorrências no âmbito da gestão de riscos de corrupção e } \\
\text { infraçes conexas } \\
\text { Divulgação das remunerações dos órgãos sociais } \\
\text { Estratégia de sustentabilidade }\end{array}$ \\
\hline
\end{tabular}

A recolha dos dados foi realizada em simultâneo com a análise de conteúdo, contribuindo para a qualidade do trabalho desenvolvido, conforme sugerem Miles, Huberman e Saldaña (2014). Após a fase da organização e codificação da informação, procurou-se explorar e interpretar os dados, relacionando-os com o problema de pesquisa. 
Esta análise utiliza o software (CAQDAS) que permite um tratamento mais sistematizado da informação. Contudo, o processo de codificação exige de forma significativa o envolvimento humano (Neuendorf, 2017) atendendo à dificuldade de automatização na identificação e interpretação dos códigos.

Em linha com Neuendorf (2017) e Krippendorff (2004), uma das preocupações principais do estudo refere-se precisamente à replicabilidade do processo, garantindo a validade e a confiabilidade dos resultados.

As investigadoras realizaram, de forma independente, a análise de duas empresas para posterior cruzamento de informação (double-check), com o objetivo de aferir a definição das categorias a avaliar, e assegurar a confiabilidade, a qualidade e a replicabilidade do modelo.

\section{CONCLUSÕES}

Até ao momento foi possível concluir que uma parte significativa das empresas públicas consideradas no estudo não divulga de forma clara toda a informação legalmente exigida nos seus sites. O acesso à informação pode ser dificultado por duas razões: (i) a navegação não é objetiva e eficiente; (ii) a informação não é apresentada autonomamente mas sim incluída noutras rubricas ou relatórios. A primeira opção dá origem a alguma perda de tempo, a procura torna-se morosa uma vez que é necessário percorrer vários menus até encontrar a informação pretendida, já a segunda pode conduzir à perda total da informação. Estas duas situações não contribuem para promover a transparência de acordo com o mencionado nos artigos $44^{\circ}$ a $47^{\circ}$ e $53^{\circ}$ e $54^{\circ}$ do Decreto-Lei n. $133 / 2013$. Uma outra constatação diz respeito à atualização da informação divulgada para os relatórios de governo societário e de sustentabilidade. Existem empresas que não apresentam relatórios há mais de 5 anos, enquanto outras falharam a apresentação nos últimos 2 anos. Da análise referente à segunda etapa do estudo, ainda a decorrer, é possível identificar no imediato um elevado grau de disparidade entre as empresas, em relação à RSE e ao impacto positivo observado nas medidas tomadas. As empresas de maior dimensão e visibilidade pública, para além de mais transparentes na divulgação da informação, têm assumido um maior número de compromissos ambientais e sociais. Assim, nesta primeira fase, as observações permitem concluir que algumas empresas do setor público não estão a contribuir para a boa implementação das políticas públicas nem para a disseminação de boas práticas nos 
domínios económico, social e ambiental, não podendo ser consideradas como um bom exemplo na divulgação destas questões.

\section{REFERÊNCIAS}

Bardin, L. (2016 [1977]). Análise de conteúdo. Lisboa: Edições70.

Bonsón, E., \& Bednárová, M. (2015). CSR reporting practices of Eurozone companies. Revista de ContabilidadSpanish Accounting Review, 18(2), 182-193.

Bovens, M., Schillemans, T., \& Hart, P. T. (2008). Does Public Accountability Work? An Assessment Tool. Public Administration, 86(1), 225-242.

Carroll, A.B. (2015). Corporate social responsibility: The centerpiece of competing and complementary frameworks. Organizational Dynamics, 44, 87-96.

COM (2011) 681 final. Communication from the Commission to the European Parliament, the Council, the European Economic and Social Committee and the Committee of the Regions: A Renewed EU Strategy 2011-14 for Corporate Social Responsibility. Brussels: Commission of the European Communities.

Faria, M.J.S. (2017). Types of financial and non-financial information disclosure about corporate social responsibility. Cadernos EBAPE, 15(9), 534-558.

Krippendorff, K. (2004). Content Analysis: An Introduction to Its Methodology (2nd ed.). Thousand Oaks, CA: Sage

Kumar, S. \& Kidwai, A. (2018). CSR disclosures and transparency among top Indian companies. International Journal of Indian Culture and Business Management, 16(1), 57-70.

Miles, M.B., Huberman, A.M., and Saldaña, J. (2014). Qualitative data analysis: a methods, 3th Ed.. Arizona State University: Sage.

Neuendorf, K. (2017 [2002]). The Content Analysis Guidebook, 2th Ed.. Thousand Oaks: Sage Publications

OECD (2018). Diretrizes da OCDE sobre Governança Corporativa de Empresas Estatais, Edição 2015, OECD Publishing, Paris.

Sánchez, R.G., Bolívar, M.P.R., \& Hernández, A.M.L. (2015). Are Australian Universities Making Good Use of ICT for CSR Reporting? Sustainability, 7, 14895-14916.

Vala, J. (1986). A análise de conteúdo. In A. S. Silva e J. M. Pinto (orgs). Metodologia das ciências sociais (pp. 101-128). Porto: Edições Afrontamento

Venturelli, A., Caputo, F., Leopizzi, R., \& Pizzi, S. (2019). The state of art of corporate social disclosure before the introduction of non-financial reporting directive: a cross country analysis, Social Responsibility Journal, 15(4), 409-423. 